\title{
Dynamics parameters estimation of an asynchronous machine plus mechanical shaft set through orbit frequency response analysis
}

\author{
F. Oliveira ${ }^{1,2}$, M. P. Donsión ${ }^{3}$, G. Peláez ${ }^{4}$ \\ ${ }^{1}$ Department of Electrical Engineering, School of Technology and Management, Polytechnic Institute of Leiria \\ Campus 2 - Morro do Lena - Alto do Vieiro, 2411-901 Leiria, Apartado 4163, Portugal \\ Phone: +351244820 300, e-mail: ftadeu@estg.ipleiria.pt \\ ${ }^{2}$ Institute for Systems and Computers Engineering at Coimbra \\ Rua Antero de Quental, Nº199, 3000 - 033 Coimbra, Portugal \\ Phone: +351239851040 \\ ${ }^{3}$ Department of Electrical Engineering \\ E.T.S.I.I., University of Vigo \\ Campus of Lagoas - Marcosende, 36310 Vigo (Spain) \\ Phone/Fax number:+0034 986 812685, e-mail: donsion@uvigo.es \\ ${ }^{4}$ Department of Mechanical Engineering, E.T.S.I.I., University of Vigo \\ gpelaez@uvigo.es
}

\begin{abstract}
This paper presents some of the results obtained upon the experimental study of the behaviour of a prototype mechanical shaft driven by an induction electric machine. The main focus of this paper will be on the mechanical response of the set, based on the measurement of a number of mechanical variables and its integration in well-known mechanical models, allowing a more accurate estimation of the actual parameters of the prototype machine. The results thus obtained can then be used to test the theoretical models, estimate mechanical parameters more accurately and generally increase knowledge on the mechanical response of the prototype set.
\end{abstract}

\section{Key words}

Mechanical vibrations, turbomachines, orbit frequency response.

\section{Introduction}

This paper presents some of the results obtained upon the experimental study of the behaviour of a prototype mechanical shaft driven by an induction electric machine.

The main focus of this paper will be on the mechanical response of the set, based on the measurement of a number of mechanical variables and its integration in well-known mechanical models, allowing a more accurate estimation of the actual parameters of the prototype machine.

The results thus obtained can then be used to test the theoretical models, estimate mechanical parameters more accurately and generally increase knowledge on the mechanical response of the prototype set.

\section{Laboratory equipment}

In order to study the influence of the electric supply to the motor on the overall behaviour of a mechanical load, a specially designed, dimensioned and built one-stage inertia flexible rotor (OSIFRO) set was used. This provided a simple mechanical load with a well-fitted (and well-known) dynamic model with which to work. Figures 1 and 2 show a view of the OSIFRO model and a representation of the rotor shaft on the bearings::

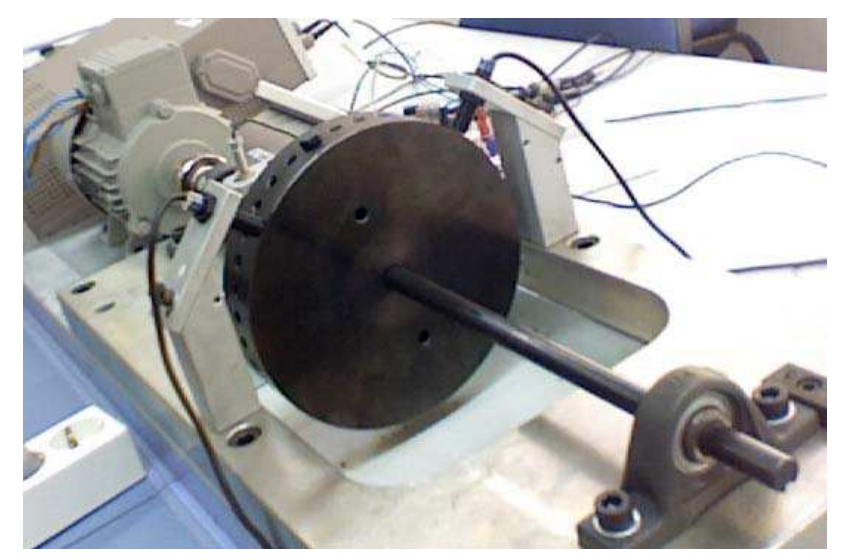

Fig. 1. A view of the OSIFRO

To drive the OSIFRO, a $0,37 \mathrm{~kW}$ squirrel-cage induction machine was connected using a three-phase input/output, $15 \mathrm{kVA}$ programmable AC power source from California Instruments, which provided perfect voltage sine waves of a range of amplitudes and frequencies. 


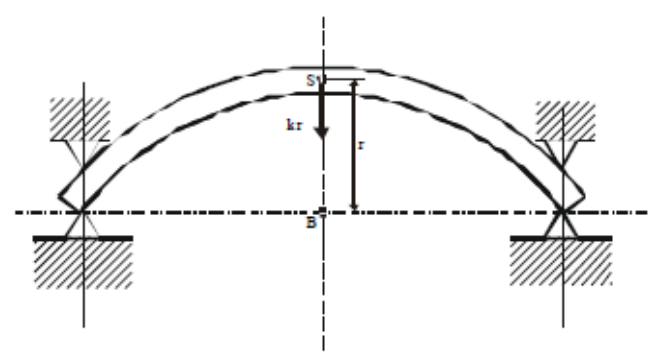

Fig. 2. A representation of the flexible rotor shaft on the bearings

The OSIFRO mechanical load is constituted by a flexible, non-linear (keyed) rotor with a cylindrical inertia. This mechanical load is intrinsically slightly unbalanced, and adequate correction masses can be added to balance the rotor.

This work required a number of electrical and mechanical quantities to be measured. In order to acquire and accommodate the several quantities to be measured, a PC-based, high-precision data acquisition card was used, along with voltage and current active probes, accelerometers, proximity inductive sensors and a keyphasor.

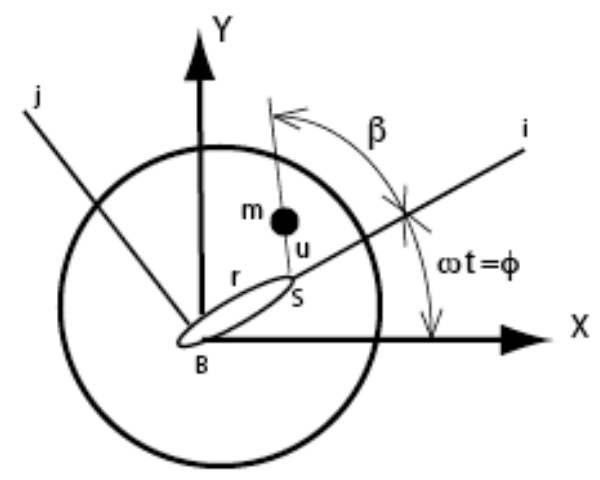

Fig. 3. One degree of freedom Jeffcot model for the flexible rotor over rigid bearings

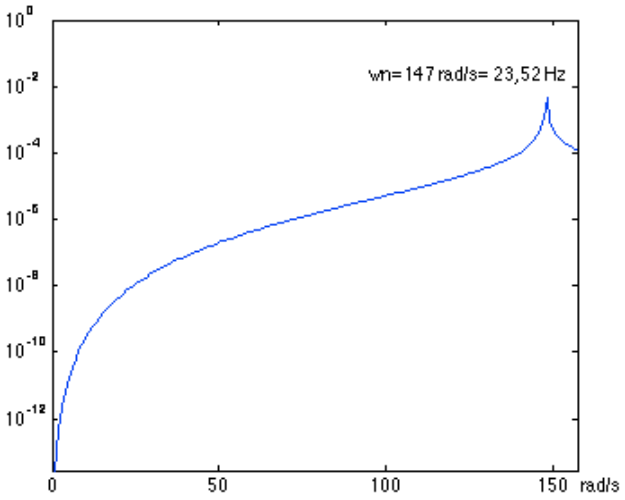

Fig. 4. Natural frequency for the OSIFRO obtained from the Jeffcot model

Figures 3 depicts the one degree of freedom Jeffcot model for the flexible rotor over rigid bearings, and figure 4 shows the corresponding modelled natural frequency.

\section{Experimental tests and results}

In order to study the behaviour of the mechanical load connected to the induction motor, a number of trials were devised and performed, and the main results are presented in this section.

Firstly, the rotor had to be balanced using the methods described in [2] and applied in [1], so that the crossing of the natural frequency of the OSIFRO would cause a lesser strain on the shaft and bearings of the machine.

With this process completed, a thorough frequency sweep was conducted, for both natural response and forced response.

Figures 5 a) to c) depict the unforced frequency response, with a starting point above the main resonance; the machine is then left to free-wheel stop, sweeping through all frequencies (including the main resonance) - (blue keyphasor signal; green and red - distance to the shaft in the $\mathrm{x}$ and $\mathrm{y}$ directions of figure 3 )

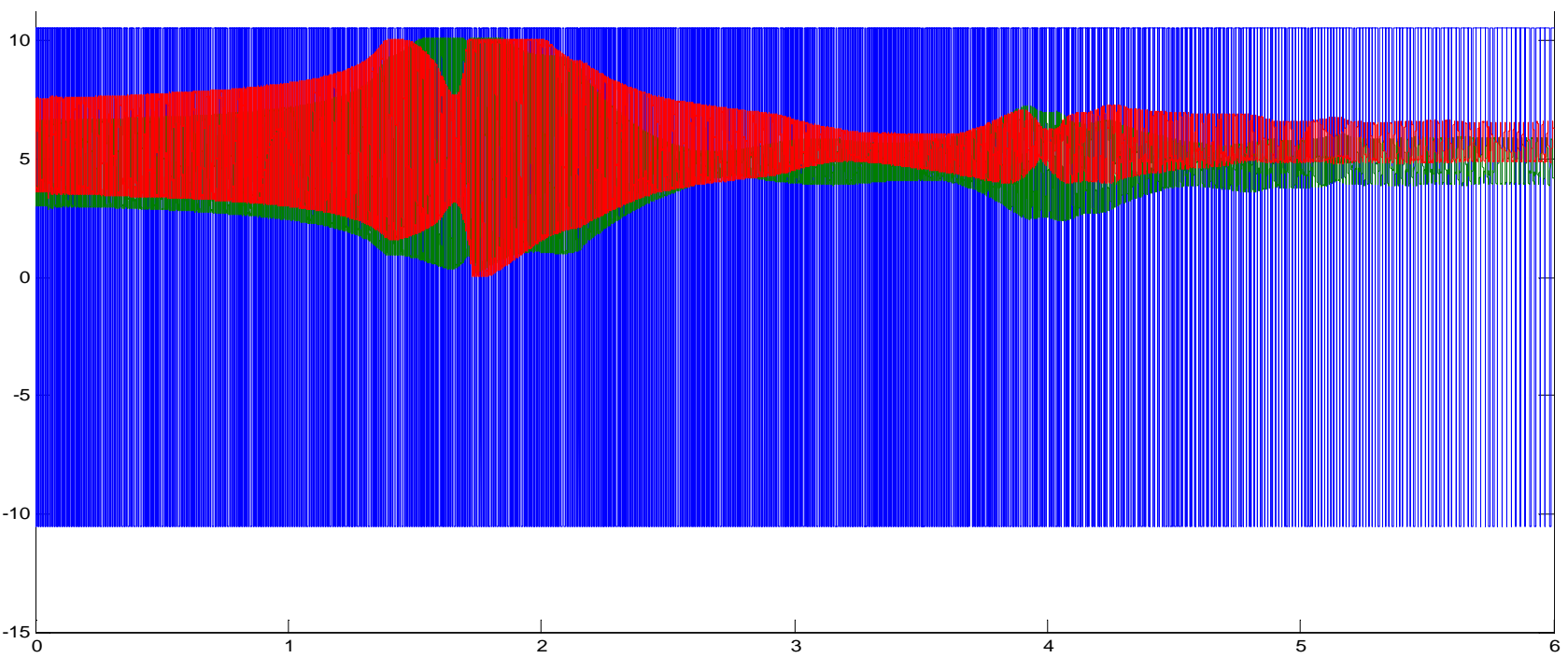

Fig. 5 a) Unforced frequency response for the OSIFRO 


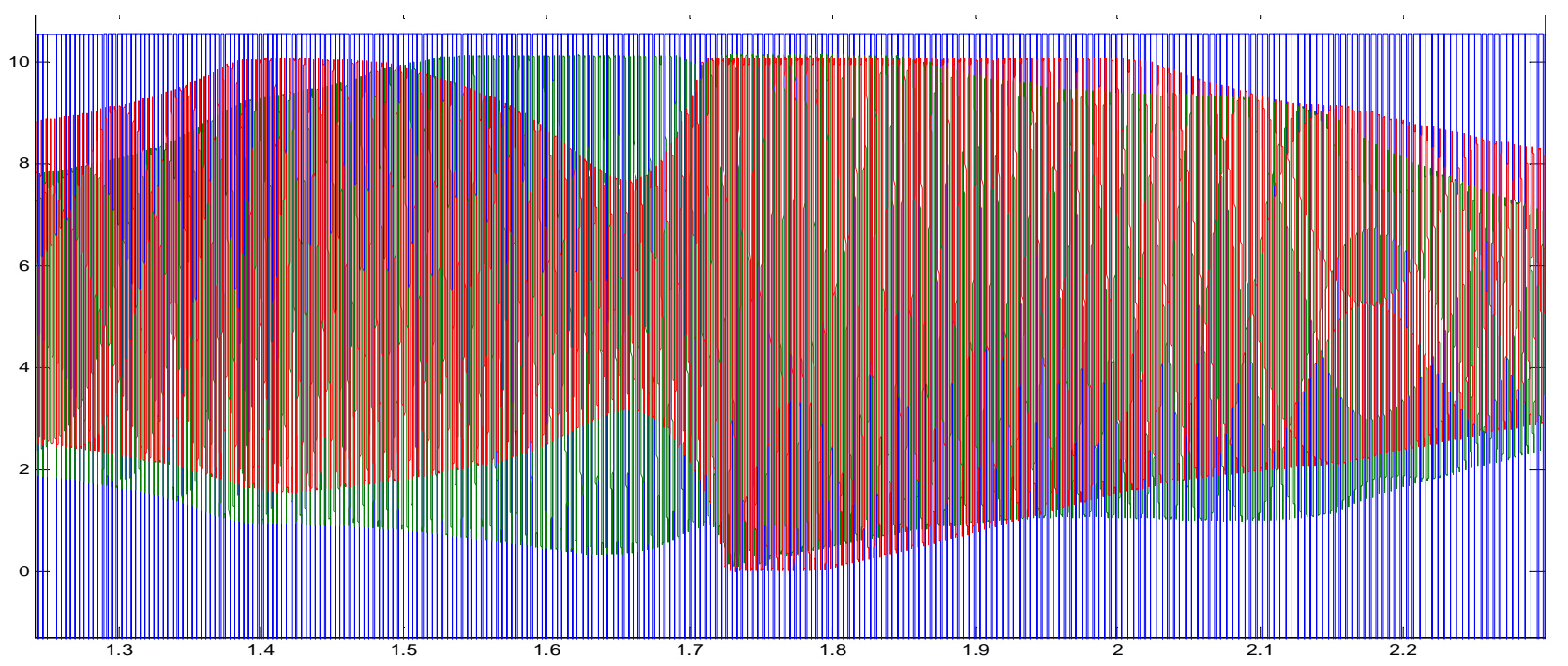

Fig. 5 b) Unforced frequency response for the OSIFRO - zoom in on the main resonance

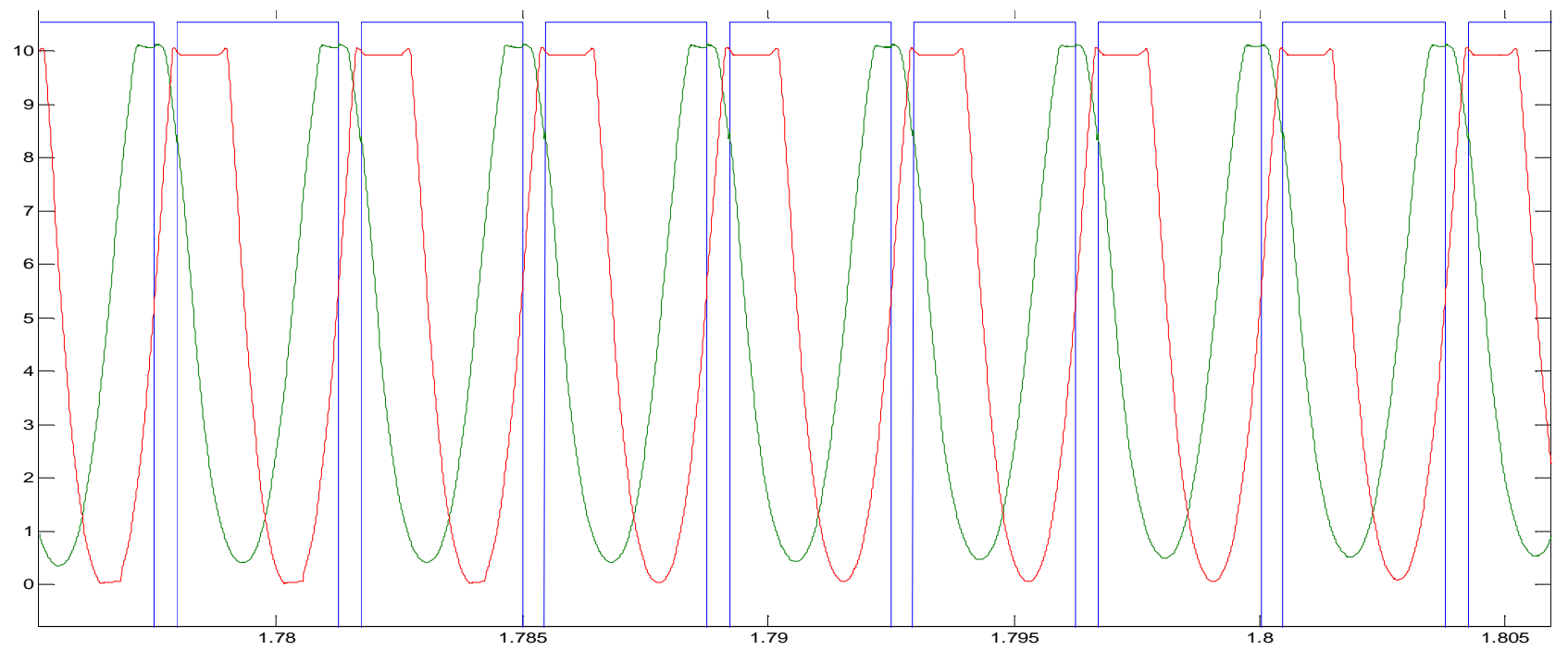

Fig. 5 c) Unforced frequency response for the OSIFRO - detail of the main resonance

The results obtained and presented in figure 5, particularly in 5 a), also show that, apart from the main resonance near the calculated natural frequency, there is another, smaller sub-harmonic resonance, which is probably due to the fact that the shaft is keyed rather than solid cylindrical. Furthermore, both resonances seem to be "double", that is, there is an overlap phenomenon in both of them, resulting in difficulty to find the actual natural frequency from the data.

An important detail is that the phase of the mechanical vibrations, i.e. the angular distance to the minimum distance to probes $\mathrm{X}$ and $\mathrm{Y}$, changes throughout the sweep, making a $>180^{\circ}$ sweep when crossing the main resonance.

Figure 6 depicts the mechanical vibrations frequency response of amplitude and phase:

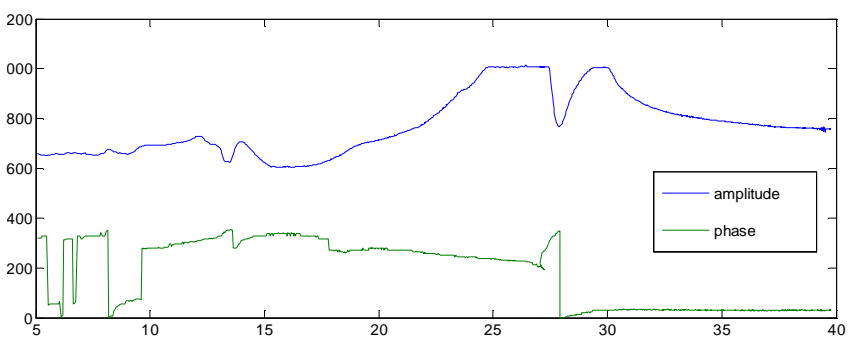

Fig. 6) Mechanical vibrations frequency response amplitude and phase

The $180^{\circ}$ crossing is also visible when an amplitudephase polar plot is sketched, evidencing the orbit as a 'trajectory', as is patent in figure 7, where the main frequency points are signalled. 


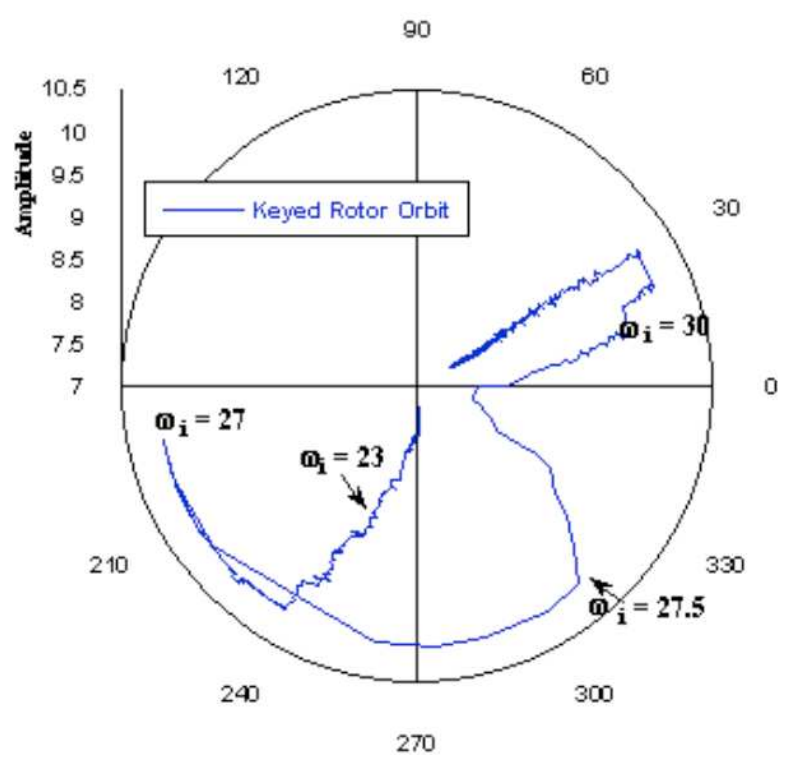

Fig. 7) Phase-amplitude polar plot of mechanical vibrations

\section{Parameter fitting}

In the following section the correlation between the theoretical and the experimental rotor orbit will be computed to extract the actual dynamic parameters from theexperimental orbit. This will allow the building a more accurate rotating machine Jeffcot model.

Since the rotor is not linear, the Jeffcot model is not expected to produce a very accurate estimation of the natural frequency and remaining dynamics parameters.

Therefore, in order to approximate more accurately, and using the fact that several characteristics are proportional in a small window, a plot was sketched to evaluate the natural frequency, which is shown in figure 8:

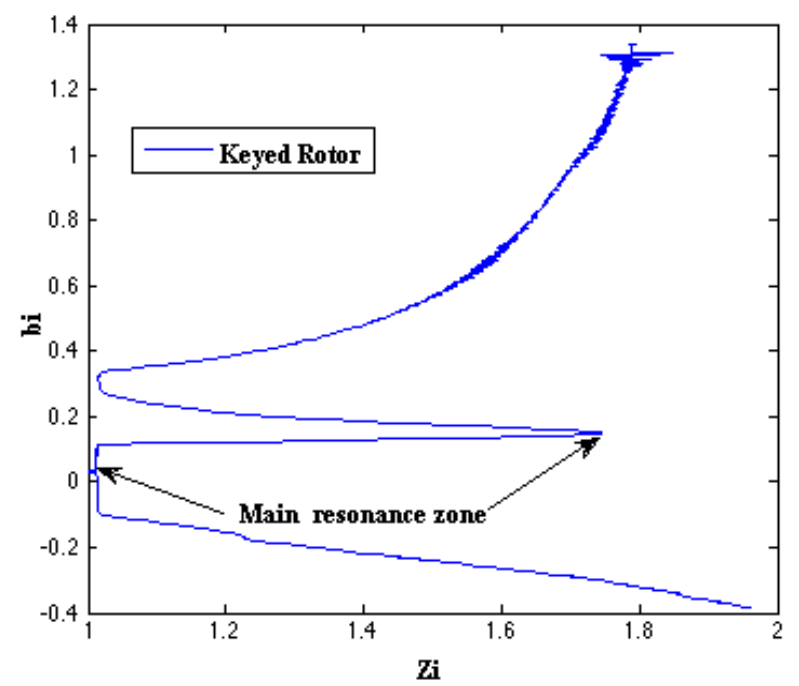

Fig. 8) Plot relating vibration amplitudes and frequencies, in order to evaluate the actual natural frequency more accurately

$\mathrm{Z}_{\mathrm{i}}$ corresponds the inverse of the fraction between observed and maximum amplitude, for each frequency (eq. 1), whereas $b_{\mathrm{i}}$ :obtained from the expected natural frequency and the remaining frequencies through (eq. 2)'

$$
\begin{gathered}
\frac{R_{i}^{2}}{R_{0}^{2}}=\frac{1}{Z_{i}} \\
b_{i}^{2}=\frac{\omega_{i}^{2}-\omega_{0}^{2}}{\omega_{0}^{2}}
\end{gathered}
$$

The parameter obtaining and mathematical manipulations are clearly out of the scope of this paper.

Applying a linear regression in the area corresponding to the main resonance, it is possible to obtain this improved estimate of the natural frequency, as depicted in figure 9:

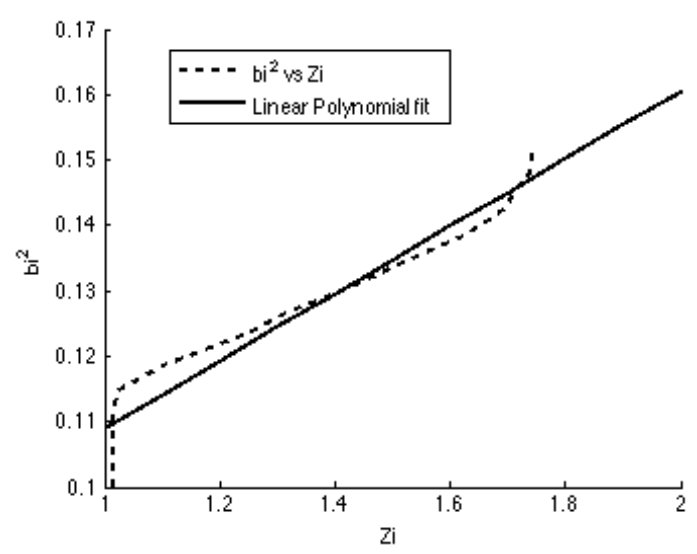

Fig. 9) Linear regression of the main resonance zone of Fig. 8

This results in a new natural frequency of

$$
\omega_{0}=\omega_{m} \frac{1}{\sqrt[4]{1+Q}}=25.30[H z]
$$

\section{Conclusions and Outlook}

In this paper a number of dynamics studies and parameters are performed, in order to improve the knowledge of the dynamic behaviour of the OSIFRO machine. The conclusions drawn and the methods used can then be also used for other machines, including larger scale machines.

In terms of the mechanical models and remaining theory that governs these processes, they were observed in most of the phenomena, though there are, as usual, some discrepancies due to model simplifications or uncertainty in some of the input parameters.

The use of the new approach referred in section 4 allowed a further improvement, allowing a relevant correction of the predicted natural frequency. 
One other point to be made is that for the global rotating machine the frequency is slightly lower due to the 'addition' of the asynchronous motor inertia to that of the mechanical shaft.

\section{Acknowledgement}

The authors wish to acknowledge the financial support of Spanish government through "Ministerio de Ciencia e Innovación" and FEDER Funds. Research project ENE2007-6803-C04-01.

\section{References}

[1] J.P. Den Hartog, "Mechanical Vibrations", MacGraw-Hill 4th ed. New York, 1956 (Reprint).

[2] G. Peláez, F. Oliveira, M. P. Donsión, "Rotor vibration reduction using rotor balancing and command shaping", Proceedings of 11 CHLIE $-11^{\text {th }}$ Spanish Portuguese Conference on Electrical Engineering, Zaragoza (Spain), 2009

[3] Childs, Dara, Turbomachinery Rotordynamics Phenomena, Modeling and Analysis, New York: WileyInterscience Publication, 1993. 